\title{
The pelled vellonodular synonvitis located as a cause of hemarthrosis in the knee
}

\begin{abstract}
Summary
Pigmented Vellonodular Synovitis (SVP) is a benign neoplasm with synovial proliferation and hemosiderin deposition, characterized by compromising large joints, especially the knee. Two clinical variants, the diffuse form (SVPD) and the localized form (SVPL), are currently described. Arthroscopic Sinovectomy and Radiosinoviortresis (RSO) are the treatment that has demonstrated better functional results. Nuclear Magnetic Resonance is a suitable method to perform the diagnosis of SVPL.
\end{abstract}

Keywords: Synovitis, Knee, Hemarthrosis

\author{
Volume II Issue I - 2019 \\ Martinez Ruiz FJ,' Salaverry Walls A, ${ }^{2}$ Bautista \\ Morales M, ${ }^{3}$ Rivera Reyes C, ${ }^{4}$ Montero \\ Garcia JG ${ }^{5}$ \\ 'Resident High Specialty Course in Joint Surgery, Mexico \\ ${ }^{2}$ Physician Assigned to the Service of Pathological Anatomy, \\ Mexico \\ ${ }^{3}$ Physician Assigned to the Area of Magnetic Resonance, \\ Neuroradiology, Mexico \\ ${ }^{4}$ Physician Assigned to the Service of Orthopedics, Deputy of \\ the Course of High Specialty in Joint Surgery, Clinic of Hip and \\ Knee, Mexico \\ ${ }^{5}$ Physician Assigned to the Service of Orthopedics, Holder of \\ the High Specialty Course in Joint Surgery, Shoulder Clinic, \\ Mexico
}

Correspondence: Felipe de Jesús Martínez Ruiz, Department of Orthopedics, Hospital Beneficencia Española de Puebla. 19 North No. 100I, Col. Jesús García, C.P. 72090 Puebla, Mexico, Email felipedj.mtzr@gmail.com

Received: October 15, 2017 | Published: January 29, 2019

\section{Introduction}

Pigmented Vellonodular Synovitis (SVP) is a benign neoplasm with synovial proliferation and hemosiderin deposition, characterized by compromising large joints, especially the knee. The prevalence of this disease is between the third and fourth decade of life. The SVP was described by Chassignac in 1852 as a nodular lesion in the sheath of a flexor tendon of the hand. ${ }^{1,2}$ In 1864, its localized form in the knee was described. In 1914, Jaffe and colleagues divided this disease into Pigmented Vellonodular Synovitis (SVP), Pigmented Vellonodular Bursitis (SVNB) and Pigmented Vellonodular Tenosynovitis (TSVP). ${ }^{2}$ Two clinical variants, the diffuse and localized form, are currently described. ${ }^{3}$

In relation to the etiology of the disease, there are authors who attribute an inflammatory character while others suggest a neoplastic origin of this entity. ${ }^{4}$ In this paper we will refer only to localized pigmented Vellonodular Pigmented Synovitis (SVPL), and it usually has a diagnosis late because the patient's symptoms are slow and progressive, presenting clinical manifestations of multiple pathologies in the knee, so it is common confusion to establish the diagnosis.

\section{Presentation of the case}

It is a male patient of 34 years of age, with no relevant disease history, with clinical signs of 4 months of evolution characterized by insidious joint effusion, on 4 occasions approximately that has been handled by several doctors and medical specialists. The main pain in the articular and suprarotullary interline, in zones 1, 6, 7 and 8 of Fulkerson. ${ }^{5}$

During physical examination, positive Apley and McMurray maneuvers were demonstrated for lateral meniscal symptomatology, positive iceberg. We performed a Nuclear Magnetic Resonance (NMR) study where thickening of the synovial membrane was observed at the expense of nodular, hypointense and heterogeneous images in the different sequences, suggestive of hemosiderin deposits, associated with this increase of synovial fluid was observed suprapatellar recess, as well as important synovitis in the medial and lateral femorotibial compartments.

After the clinical integration with the cabinet studies, a diagnostic and therapeutic arthroscopy of his right knee was proposed, where severe synovial infiltration with hypertrophy and hyperpigmentation was observed; the macroscopic characteristic of the synovial was papillary and with brown pigmentation, an extensive subtotal synovectomy and haemarthrosis drainage were performed. The histopathological study of the samples obtained reported erythrophagocitosis, findings compatible with pigmented vellonodular synovitis, derived from these results the patient was channeled to the Oncology service for Radiation Sinovectomy.

\section{Discussion}

SVP is a joint pathology that results in synovial hypertrophy with diffuse haemosiderin deposits that are not calcified; can affect any joint, bursa or tendon sheath. They are usually divided according to the site of origin in patterns (intraarticular or extra-articular) and growth (localized or diffuse). The term "Pigmented Vellonodular Synovitis" is generally used when diffuse intraarticular involvement is present, as is the case of our patient. ${ }^{1,5}$

Several imaging methods have been used for the diagnosis of SVPL. These methods include simple x-ray, Computed Axial Tomography (CT) and MRI, of which the latter has been shown to be the noninvasive method of choice for the diagnosis of the disease, since it allows the extension of the disease to be determined and helps differentiate the localized form from the diffuse. In the first form, a periarticular or synovial mass with varying degrees of bone erosion can be observed. The high content of hemosiderin allows it to be seen as a low signal or speckled lesion in T1 and T2. In the diffuse form, a poorly delimited mass or significant synovial thickening is observed, 
and the dark image in the dark has been classically described in both $\mathrm{T} 1$ and $\mathrm{T} 2{ }^{6}$

Synovial biopsy often reveals the definitive diagnosis, and this can be done by arthroscopy or open arthrotomy depending on the location and extent of the disease. In the pathology, a reddish-brown thickened synovial membrane due to hemosiderin deposition can be observed with numerous villus-like projections in conjunction with multinucleated giant cells and histiocytes. Some conditions that cause hemiarthrosis such as hemophilia, hemochromatosis and hemosiderosis may present hemosiderin in biopsies; however, the pigment is confined to synovial cells and macrophages whereas in the SVP it is diffusely located. Malignant transformation is extremely rare, but has been reported in the literature.?

Although the abundant synovitis can be removed through arthroscopic surgery, there are reports in the literature of recurrence between 8 and $46 \%$. Therefore, after 6 weeks of surgery, once the wound is healed, a sinusectomy must be performed. Radiation, also called Radiosinoviortresis (RSO). ${ }^{8}$ Kat et al. ${ }^{9}$ report a $100 \%$ success rate when combining surgical synovectomy and RSO (Figures 1-11).
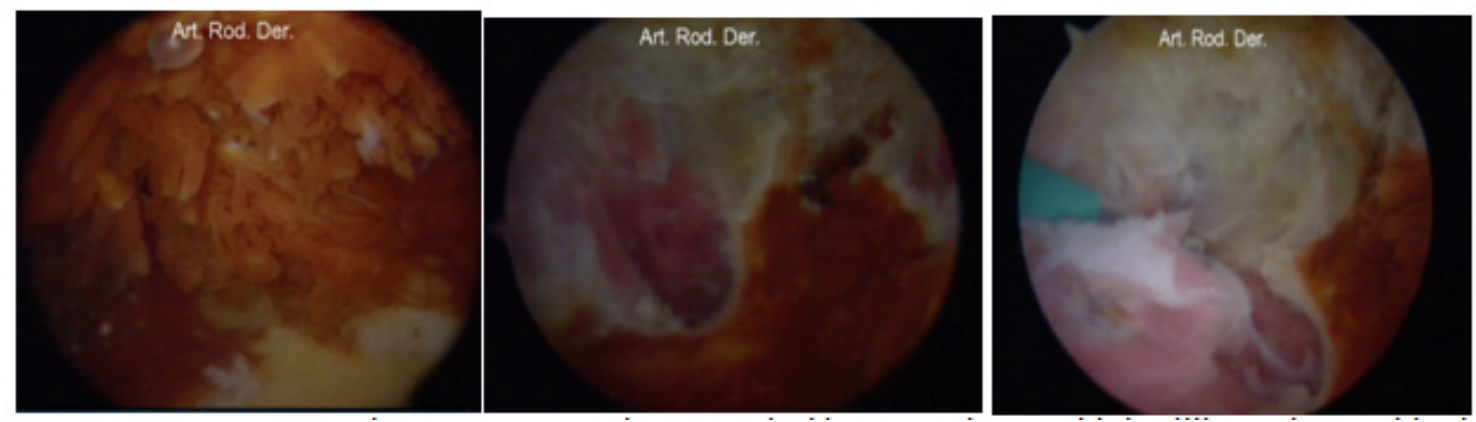

Figure I, 2 \& 3 Knee arthroscopy. Pre- and post-surgical images, where multiple villi are observed in the synovial intra-articular diffuse reddish-brown color.

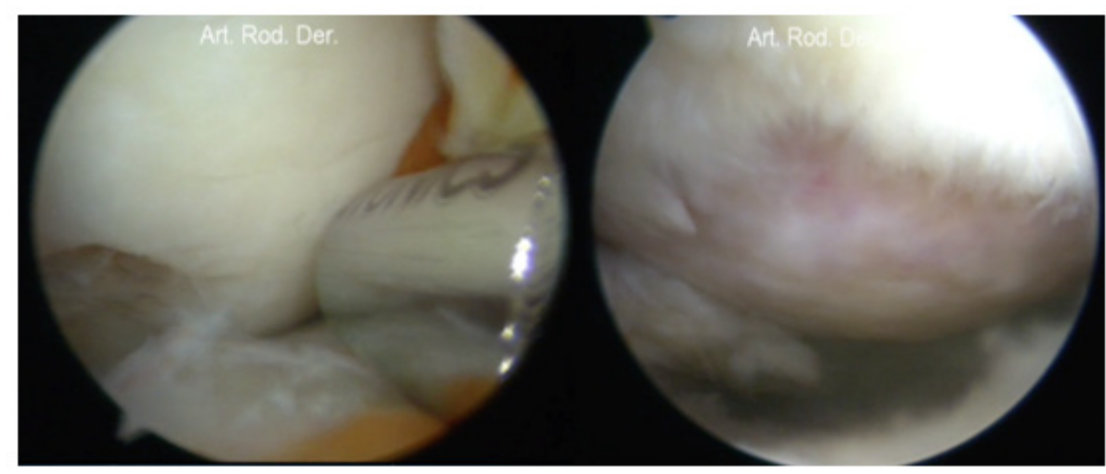

Figure 4\&5 Knee arthroscopy. Chondral lesions and exposure of the subchondral bone are observed, with hyperpigmentation of diffuse distribution at the level of the femoral condyles.
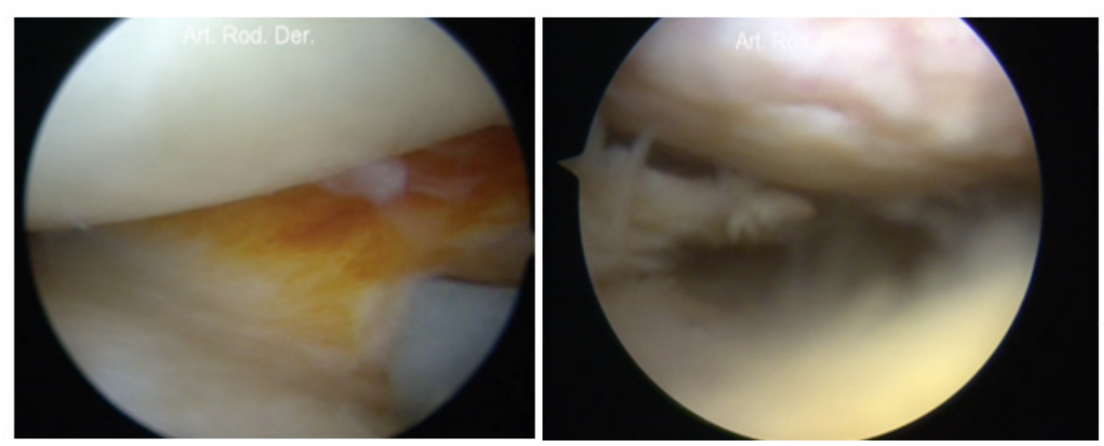

Figure 6 \& 7 Knee Arthroscopy. It is observed infiltration and pigmentation in capsulomeniscal junction of the lateral compartment, as well as injury of the medial meniscus of the internal compartment.

Also, in a meta-analysis, Kresnik et al..$^{10}$ demonstrated favorable results until they ranged from $77 \pm 23.5 \%$ when the combination of both procedures were performed in patients with Pigmented Vellonodular Synovitis. However, pregnancy contraindications should be considered as absolute, cutaneous infections, broken Baker's cyst, since complications, although not so frequent, are described in the literature up to $2 \%$, and may be local radionecrosis, septic arthritis , thrombosis, cartilage damage and risk of malignization, mainly. In 2006, a nationwide survey in Germany, Kampen et al. ${ }^{11}$ investigated the different nucleotides currently used, demonstrated that RSO is associated with complications in only about 1 in 1000 cases. 


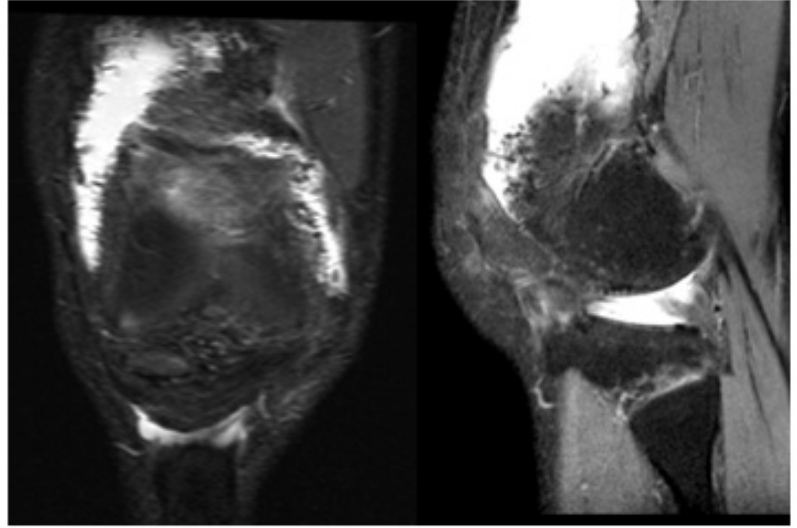

Figure 8 Coronal and sagittal T2 images with fat suppression. Areas with absence of signal within a joint effusion in both compartments of the knee. It is observed a heterogeneous collection dependent on synovium, with a diffuse intraarticular distribution due to hemosiderin deposition.

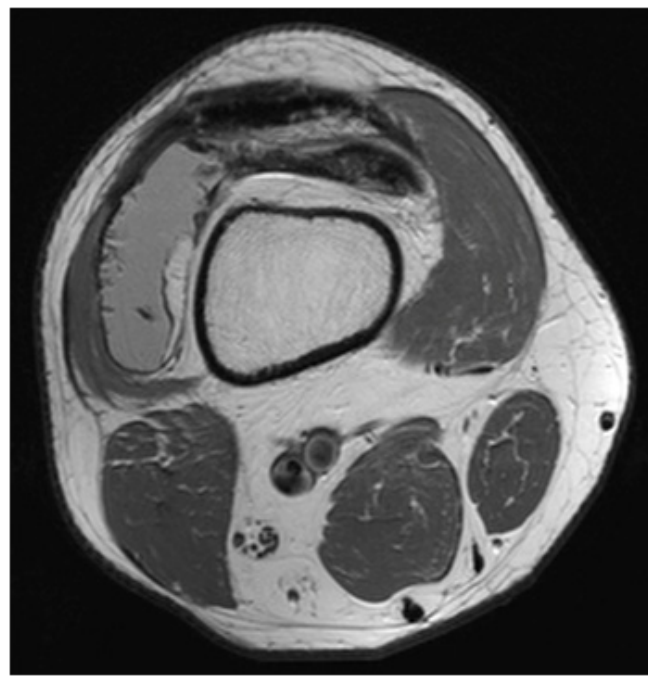

Figure 9 Axial image obtained with proton density technique.A heterogeneous collection with a marginal hyper-intense component is observed within a collection of lower signal intensity in the lateral compartment. Small peripheral defects with absence of signal on the walls of this collection.

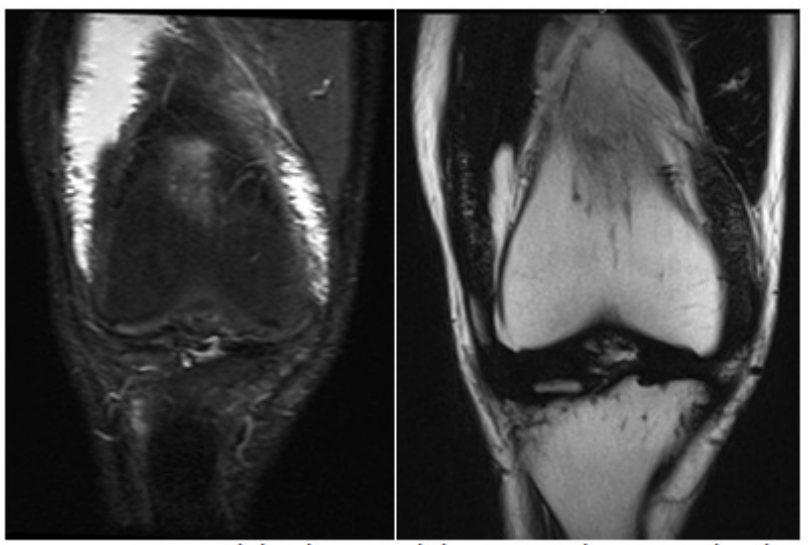

Figure 10 T2-weighted coronal images and proton density. Heterogeneous collection is shown in both compartments. Peripheral defects with absence of signal within both collections.

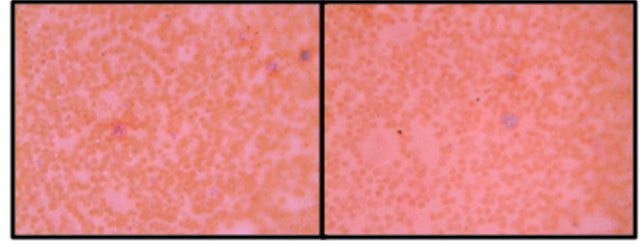

Figure I I Micrographs of synovial biopsy. Abundant erythrocytes and some macrophages with diffuse erythrophagocytosis.

\section{Conclusion}

Localized Diffuse Vellonodular Synovitis is a rare condition, with a catastrophic prognosis if its diagnosis is not made in a timely manner. With the advent of new surgical and medical techniques, the arthroscopic synovectomy in combination with Radiation Sinovectomy or also called Radiosinoviortresis (RSO) has proven to be an effective treatment with a high rate of good results, and with a low relapse. On the other hand, Nuclear Magnetic Resonance is an adequate method to perform the SVPL diagnosis, since it allows to determine the location and extent of the lesions, and postoperative magnetic resonance studies can determine the stability of the disease or its recurrence.

\section{Conflicts of interest}

Authors, their immediate family members, and the research centers with which they are affiliated have received no financial or other benefits from any commercial entity related to the subject matter of this article.

\section{References}

1. Goldman A, DiCarlo E. Pigmented villonodilar synovitis diagnosis and differential diagnosis. Radiol Clin North Am. 1988;26:1327-1347.

2. Restrepo JP, Molina MP. Sinovitis Villonodular pigmentaria. Revisión de la literatura a propósito de un caso. Rev Colombiana Reum. 2010;17(2):132-135.

3. Murphey M, Rhee J, Lewis R, et al. Pigmented villonodular synovitis: radiologic-pathologic correlation. Radiographics. 2008;28(5):14931523 .

4. Makino A, Musculo L, Costa-Paz M, et al. Sinovitis vellonodular pigmentada localizada de rodilla: diagnóstico con resonancia magnética y su tratamiento artroscópico. Rev Asoc Arg Ortop y Traumatol. 1997;62(2):257-263.

5. Warren H, Ortiguera C, Raouf E. Pigmented villonodular synovitis Radiographics. 2008;28(5):1519-1523.

6. Tyler W, Vidal A, Williams R, et al. Pigmented villonodular synovitis. $J$ Am Acad Orthop Surg. 2006;14(6):376-385.

7. Kalil R, Unni K. Malignancy in pigmented villonodular synovitis. Skeletal Radiol. 1998;27(7):392-395.

8. Mödder Gynter. Radionuclide Therapy of Inflammatory Joint Diseases (Radiation Synovectomy, Radiosynoviorthesis). Therapeutic Nuclear Medicine. 2013;459-493.

9. Kat S, Kutz R, Elbracht T, et al. Radiosynovectomy in pigmented villonodular synovitis. Nuklearmedizin. 2000;39(7):209-213.

10. Kresnik E, Mikosch P, Gallowitsch HJ, et al. Clinical outcome of radiosynoviorthesis: a meta-analysis including 2190 treated joints. Nuclear Med Commun V. 2002;23(7):683-688.

11. Kampen WU, Matis E, Czech N, et al. Serious complications after radiosynoviorthesis - survey on frequency and treatment modalities. Nuklearmedizin. 2006;45(6):2006-2268. 